

\section{Das Plus macht den Unterschied}

Kuraray Noritake stellt den neuen PANAVIA SA Cement Plus vor, den Nachfolger von PANAVIA SA CEMENT. Vor mehr als 30 Jahren kam PANAVIA als der 1 . Befestigungszement mit MDP-Monomer auf den Markt. Der neue Zement kann bei Raumtemperatur (unter $25^{\circ} \mathrm{C}$ ) gelagert werden und vereinfacht so die Aufbewahrung. Aber auch die Handhabung hat sich wesentlich verbessert, die Ausbringung des Materials kann schon mit wenig Druck vorgenommen werden.

\section{Zahnerhaltung}

\section{Vom Praktiker für}

\section{den Praktiker}

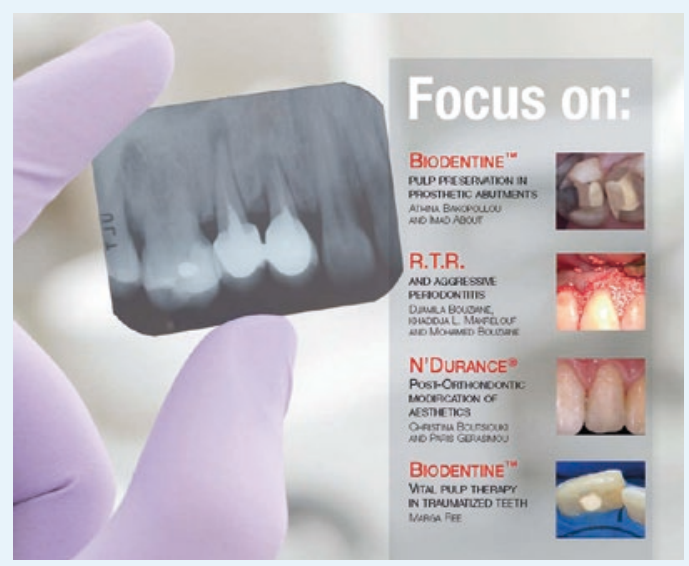

Biodentine von Septodont fördert die regenerative Dentinogense und stimuliert Pulpazellen. Reiz- und Reparaturdentin sowie Dentinbrücken werden schneller gebildet und schaffen die notwendige Voraussetzung für eine optimale Pulpaheilung. Die dem menschlichen Dentin ähnlichen mechanischen Eigenschaften machen das Produkt so vielseitig einsetzbar im Bereich der KalziumsilikatZemente.

Zur Förderung des Erfahrungsaustauschs unter Zahnmedizinern hat Septodontin 2012 die Case Studies Collection (CSC) etabliert, die internationale Fallberichte umfasst. Im Fokus stehen SeptodontProdukte, die weltweit Vertrauen genießen. Aktuell ist die Ausgabe 8 erschienen, Download unter www.septodont.de

Nach einer Pressemitteilung der

Septodont GmbH, Niederkassel

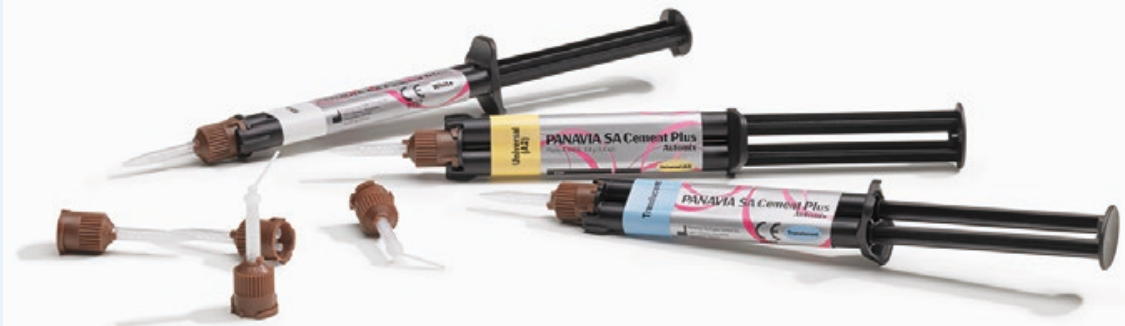

Die um die Farbe Transluzent erweiterte Farbpalette bietet jetzt noch ästhetischere Ergebnisse. Aber auch in seiner erhöhten Haftkraft findet das Plus im Namen seine Berechtigung. Aufgrund der in dem Produkt enthaltenen MDP-

Monomer und Dual-Cure-Technologie bietet PANAVIA SA Cement Plus eine einfache und zuverlässige Lösung.

Nach einer Pressemitteilung der Kuraray Europe GmbH, Hattersheim Internet: www.kuraray-dental.eu

\section{Implantologie \\ Mehr zahnlose Patienten besser versorgen}

Das All-on-4-Konzept für die Rehabilitation zahnloser Kiefer hat viele Vorteile für die Patienten: Sie erhalten auf nur 4 Implantaten einen festsitzenden implantatgetragenen Zahnersatz, der in der Regel sofort belastet werden kann. Augmentative Maßnahmen sind nicht notwendig. Wie das Konzept „Feste Zähne an einem Tag" erfolgreich in der Praxis umgesetzt wird, haben die beiden All-on4-Spezialisten Dr. Bernd Quantius und Dr. Ana Ferro, Maló-Klinik Lissabon, mit ZTM Wolfgang Sommer am 6. September in Mönchengladbach gezeigt.

Auf dem Programm standen eine Live-OP und die Sofortversorgung zweier Patienten mit der provisorischen Brücke. So konnten die 9 Teilnehmer den Ablauf der All-on-4-Behandlung von der Extraktion der Restbezahnung über die Insertion der 4 Implantate pro Kiefer bis hin zum Einsetzen der provisorischen Brücke in Echtzeit verfolgen. Besonders positiv kam bei den Zahnärzten an, dass sich aufgrund der limitierten Teilnehmerzahl schnell ein intensiver Fachaustausch mit den beiden Spezialisten entwickelte. Sie gehören zu den Top-Referenten für die Methode nach Prof. Paulo Maló. Dr. Quantius ist in Deutschland der 1. Zahnarzt, der offizieller Partner der Maló-Klinik in Lissabon ist, und er gehört zu den insgesamt 18 All-on-4-Kompetenzzentren von Nobel Biocare in Deutschland. Dr. Ferro von

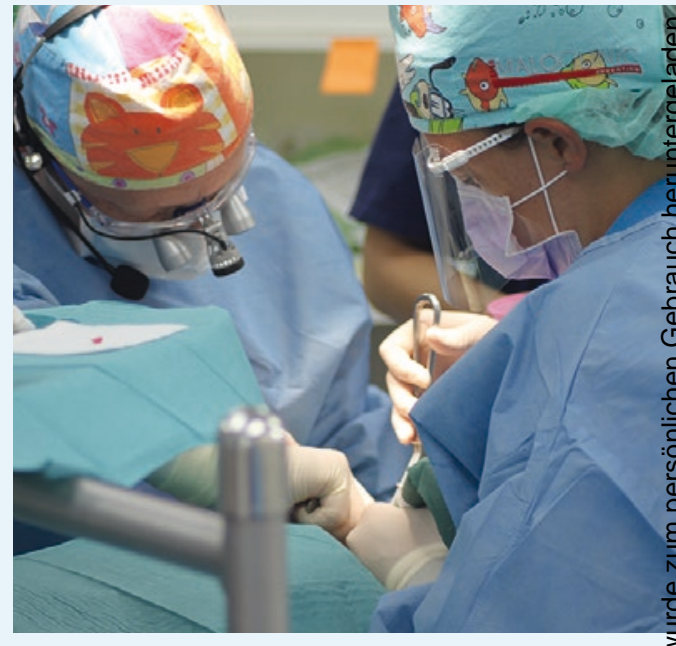

In der Live-OP zeigten Dr. Quantius und Dr. Ferro, wie die angulierten Implantate für das All-on-4-Konzept gesetzt werden.

der Maló-Klinik ist weltweit eine der TopReferentinnen für das Thema All-on-4.Ein besonderes Highlight ist der nächste Kurs im Mai/Juni 2015 in Mönchengladbach. Dann wird als Referent Prof. Paulo Maló erwartet, der Entwickler des Konzepts. Da die Teilnehmerzahl begrenzt ist, können sich Interessierte bereits jetzt unter fortbildung@nobelbiocare.com vorregistrieren lassen.

Nach einer Pressemitteilung der Nobel Biocare Deutschland $\mathbf{G m b H}$, Köln Internet: www.nobelbiocare.com 
Chirurgie

Erweitertes Portfolio:

Das Beste neu definieren

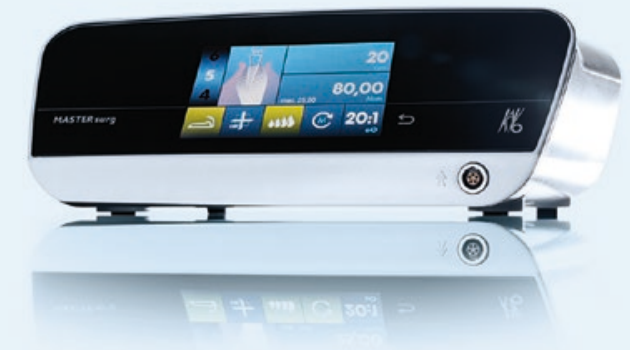

Das neue KaVo MASTERsurg LUX Wireless-Chirurgiegerät ergänzt das KaVo Chirurgie-Portfolio in idealer Weise. Die Chirurgieeinheit überzeugt durch die einfache, intuitive Programmierung über das fugenlose Touch-Display mit hygienefähiger Glasoberfläche. Der kabellose Funk-Fußanlasser bietet Anwendern die gewünschte Bewegungsfreiheit am Behandlungsfeld und eine hohe Flexibilität. Die grafische Echtzeitanzeige von Drehmoment und anderen Parametern auf dem Display unterstützt die Behandlungsabläufe. Wichtige digitale Daten können zu Dokumentationszwecken einfach gespeichert werden.

Der neue INTRA LUX S600 LED, einer der leichtesten und kleinsten Chirurgiemotoren, liefert höchste Leistung und Präzision. Die One-Touch-Auto-Kalibrierung ermöglicht präzise Drehzahlen und präzise Drehmomente für höchste Sicherheit. In Sachen Leistung und Komfort setzt KaVo mit den EXPERTsurg- und den MASTERsurg-Chirurgiegeräten, den SURGmatic-Chirurgieinstrumenten und dem INTRA LUX S600 LED-Motor auf hohe Leistung und Präzision, das Implantologen und Kieferchirurgen in ihrer täglichen Arbeit unterstützt.

Nach einer Pressemitteilung der

KaVo Dental GmbH, Biberach/ Riss
Jubiläum

\section{Jahre \\ Bissregistrat}

Das Bissregistrat Futar der Kettenbach $\mathrm{GmbH} \&$ Co.KG feiert 20-jährigen Geburtstag. Es zeichnet sich als schnellabbindendes, additionsvernetzendes Bissregistriermaterial auf Vinylpolysiloxan-Basis aus. Seit der Einführung vor 20 Jahren wurde das Futar-Portfolio sukzessive zu einer Familie mit 6 Produkten ausgebaut. So findet sich für die verschiedenen Aufgabenstellungen und Präferenzen das spezifische Material. Ob besonders kurze Mundverweildauer, extra lange Verarbeitungszeit, Scanbarkeit, extrem hohe Endhärte oder Fräsbarkeit - für jede Anforderung hat Kettenbach das entsprechende Futar-Produkt entwickelt.

Nach einer Pressemitteilung der Kettenbach GmbH \& Co. KG, Eschenburg Internet: www.kettenbach.de

\section{CAD/CAM}

\section{Neues aus Finnland}

Das CAD/CAM-Konzept von Planmeca ist nachvollziehbar. Planmeca selbst verfügt über CAD / CAM-Kompetenz in Form von Hard- und Software. Langjähriges Fräs-Know-how kaufte man sich durch die Übernahme von E4D Technologies ein und vernetzte alles mit existierenden Planmeca-Produkten. Die Basis bildet beispielsweise die Romexis-Software, die nicht nur Röntgen und DVT, sondern jetzt auch CAD/CAM managt. Das hat u.a. den positiven Nebeneffekt, dass Modelle (Abdrücke und Gipsmodelle) im DVT gescannt werden und die Daten nahtlos weiterverarbeitet werden können. Eine weitere Komponente ist der Intraoralscanner. Der PlanScan besitzt austausch- und autoklavierbare Scannerspitzen. Drei Größen werden angeboten und blaues LED-Licht ersetzt den Puder. Ist die Restauration mit der Designsoftware PlanCAD Easy konstruiert, startet die 4-achsige PlanMill 40 ihre Arbeit. Sie kann provisorischen und definitiven Zahnersatz aus Kunststoff, Glaskeramik, Zirkoniumdioxid oder auch Hybridkeramiken herstellen. Die Gerätemodule

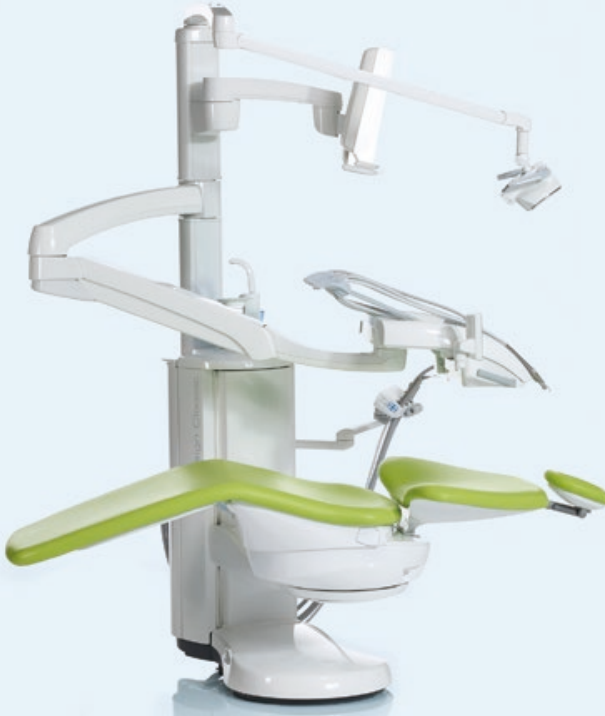

des bereits lieferbaren Chairside-Systems können nach Wunsch einzeln oder als Komplettlösung geordert werden. $\mathrm{Ab}$ Ende des Jahres wird zusätzlich ein Labside-System angeboten. Streifenlichtscanner, eine Designsoftware für die Konstruktion von Abutments, Suprastrukturen, Stegen, Schienen und Modellen sowie ein 5-achsiges Trocken- und Nassfräsgerät mit Blöcken und Ronden werden damit den Laboralltag vereinfachen. Mit einer Deutschlandpremiere

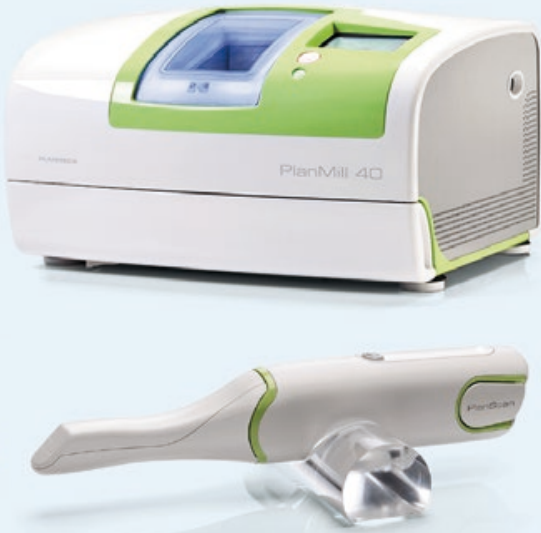

startet Planmeca in die Herbst-Fachdental-Saison. Die neue Behandlungseinheit Sovereign Classic ist sofort als PlanmecaEinheit zu erkennen. Eine Stärke der neuen Einheit lässt sich mit dem Begriff intuitiv umfassen: von den Bedienungselementen als Touchscreen-Display, über die das modernste Infektionsschutzsystem bedient wird, bis hin zum barrierefreien Rechts-/Linkshänder-Tausch. Für die IDS 2015 kündigt Planmeca bereits weitere Neuheiten an.

Nach einer Pressemitteilung der Planmeca Oy, Helsinki/Finnland

Internet: www.planmeca.com/ 


\section{Für den Espresso-Genuss!}

Die Praxis-Collection von Dürr Dental trägt mit edlem Porzellan der Marke Kahla in vielen Praxen zu einem sympathischen Ambiente bei. In diesem Herbst hat sich das Unternehmen wieder für einen ganz besonderen Porzellanartikel entschieden: Espressotassen mit samtweicher Oberfläche aus der Five Senses Touch!-Designreihe von Kahla. In Anlehnung an dieses logische Farbsystem sind auch die Tassen in Blau, Gelb, Rosa und Grün erhältlich. Vier 2,5-LiterFlaschen Dürr System-Hygiene und ein

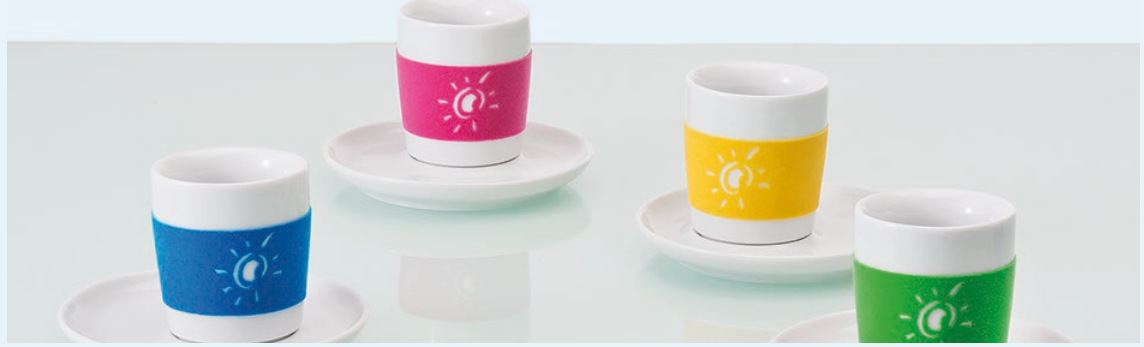

2er-Set Kahla Touch!-Espressotassen können bis 31. Dezember 2014 als Aktions-Paket beim dentalen Fachhandel bestellt werden. Das Angebot ist gültig in Deutschland und Österreich.

Nach einer Pressemitteilung der DÜRR DENTAL AG, Bietigheim-Bissingen

Internet: www.duerrdental.com

\section{Chirurgie}

\section{Arzneimittelzulassung für individuelle Knochenblöcke erteilt}

Zimmer Dental erweitert sein umfangreiches regeneratives Produktsortiment um eine weitere Option und gibt die Markteinführung des Puros Allograft Patientenindividuellen Blocks in Deutschland bekannt. Basierend auf dreidimensio-

\footnotetext{
* Hersteller und Inhaber der Arzneimittelzulassung ist die Tutogen Medical GmbH. Mitvertreiber und pharmazeutischer Unternehmer ist die Zimmer Dental GmbH.
}

nalen Röntgendaten des Defektbereichs wird mittels CAD/CAM-Technologie ein patientenindividueller Knochenblock angefertigt. Der Puros Allograft Patientenindividuelle Block ist seit Juni 2014 als Arzneimittel unter der Zulassungsnummer PEI.H.04761.01.1 zugelassen* und ab Oktober 2014 verfügbar.

Für weitere Informationen über Puros Allograft Patientenindividuelle Blöcke wenden Sie sich an einen Vertriebsmitar-

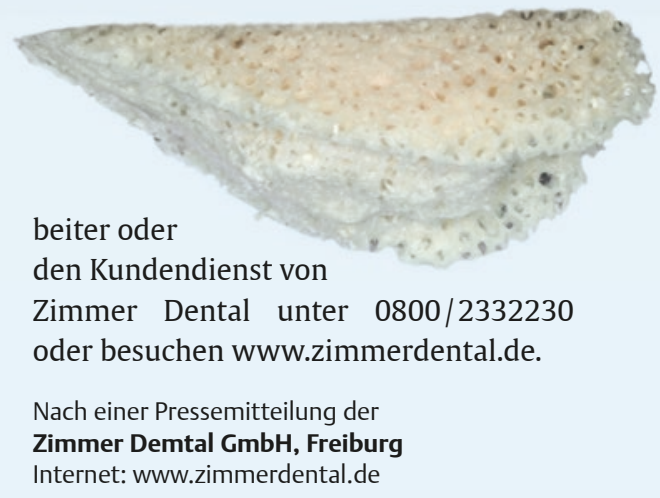

\section{Neues temporäres Befestigungsmaterial für Provisorien}

Bifix Temp ist das neue temporäre Befestigungsmaterial für Provisorien von VOCO. Die transluzente, zahnähnliche Farbe Universal passt sich Provisorien ideal an, schimmert nicht durch und unterstützt das natürliche Aussehen der temporären Versorgung. Dank Filmdicken von unter $5 \mu \mathrm{m}$ können Provisorien passgenau und ohne aufwendige Nachbearbeitung befestigt werden. Ein weiterer Vorteil ist die durch die KompositBasis unterstützte Dualhärtung. Das Material ist für ca. 90 s verarbeitbar und bindet durch Selbsthärtung innerhalb von 4 min ab. Durch den Einsatz einer Polymerisationslampe kann die Abbindezeit verkürzt werden. Der Zahnarzt ist somit flexibel. Auch für die Überschussentfernung kommt hier die sogenannte „Tack-Cure“-Technik zum Einsatz, d.h.

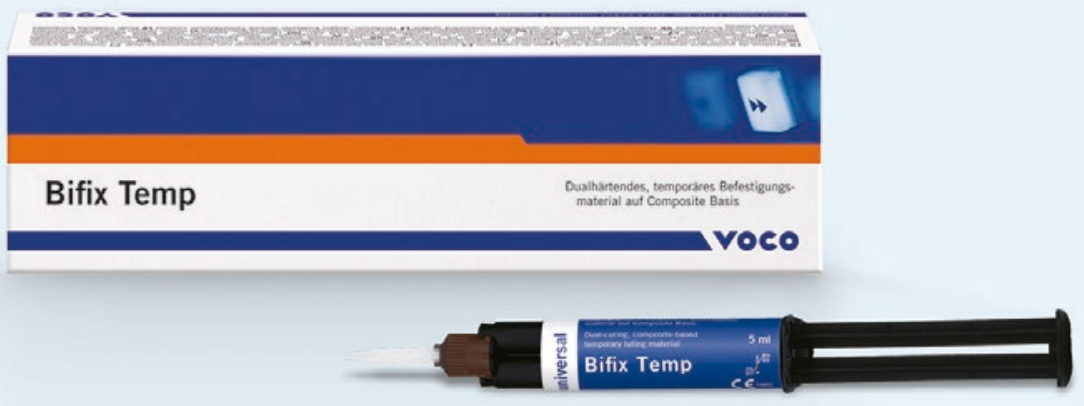

Überschüsse werden durch kurzes Belichten in eine elastische Konsistenz gebracht und bequem in einem Stück entfernt. Eventuelle Rückstände lassen sich aufgrund der universellen Farbgebung sehr gut auf der Gingiva erkennen und entfernen. Die Applikation ist durch die bewährte QuickMix-Spritze mit der Mischkanüle Typ 9 sehr einfach, da die
Komponenten beim Austragen automatisch gemischt werden. Durch die kurze Kanüle ist der Materialverlust gering und es können dadurch mehr Anwendungen pro Spritze durchgeführt werden.

Nach einer Pressemitteilung der voco GmbH, Cuxhaven

Internet: www.voco.de 


\section{Schnelle Hilfe im neuen FAQ-Bereich}

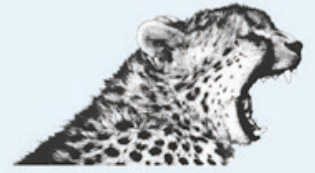

C H A R L Y

Das Prinzip Vorsprung

$\mathrm{Zu}$ den regionalen Dentalmessen in diesem Herbst baut die solutio $\mathrm{GmbH}$ ihr Serviceangebot aus. Im neuen FAQ-Bereich auf der Internetseite des Unternehmens finden CHARLY-Anwender ab Mitte Oktober Antworten auf häufig gestellte Fragen rund um die PraxismanagementSoftware. Besucher können einen Blick auf die FAQs werfen und sich von den Vorteilen des neuen Website-Bereichs überzeugen. Die solutio $\mathrm{GmbH}$ greift hier die am häufigsten gestellten Fragen in der Hotline und dem technischen Support auf und beantwortet diese - direkt und ohne Wartezeit für die Kunden. Außerdem werden die FAQs stets erweitert und aktualisiert. Mit Funktionen wie der befundorientierten Behandlungsplanung, der Prozessorientierten Abrechnung (P.O.A.) und einem transparenten Controlling sorgt das Programm für mehr Umsatz und mehr Möglichkeiten, Zahnarztpraxen erfolgreich zu managen. Das prozessorientierte Programm erfasst alle vom Zahnarzt erbrachten Leistungen lückenlos und rechnet diese centgenau ab. Dank übersichtlicher Terminplanung im Programm und optimierten Praxisabläufen amortisiert sich CHARLY durchschnittlich bereits nach circa 2 Jahren (07.11.2014 - 08.11.2014, iddeutschlandmitte Frankfurt, Halle 5.1, Stand F11).

Nach einer Pressemitteilung der

solutio GmbH - Zahnärztliche Software und

Praxismanagement, Holzgerlingen

Internet: www.solutio.de

\section{Internet}

\section{Der neue eShop ist online}

Mehr Service und ein neues Design - der neue Straumann eShop präsentiert sich in einem völlig anderen Format. Den Besucher erwarten eine verbesserte Navigation inklusive Produktvorschläge, eine laut Straumann intuitivere Bedienung sowie zahlreiche neue Funktionen. Jeder Onlinekunde hat die Option, sich ein individuelles Benutzerkonto anzulegen. Damit erhält er unter anderem die Möglichkeit, seine Bestellhistorie anzuschauen, Bestellvorlagen anzulegen oder Rechnungen online aus- zudrucken. Ab sofort kann der Käufer auch mit der Bezahlmethode „Kreditkarte“ sicher und bequem zahlen. Jeden Monat erscheinen neue exklusive Produktangebote oder besondere Onlineaktionen auf der Startseite des eShops. Zur Einführung übernimmt Straumann bis zum 31. Dezember 2014 die Standard-Versandkosten bei einem Bestellwert ab $250 €$.

Nach einer Pressemitteilung der Straumann GmbH, Freiburg

Internet: www.straumann.de/eshop 